\title{
Évaluation des pratiques agricoles des légumes feuilles : le cas des utilisations des pesticides et des intrants chimiques sur les sites maraîchers de Ouagadougou, Burkina Faso.
}

\author{
Tarnagda Bakary ${ }^{1}$, Tankoano Abel ${ }^{1}$, Tapsoba François ${ }^{1}$, Sourabié Pane Bernadette ${ }^{2}$, Abdoullahi Hissein \\ Ousman', Djbrine Adoum Oumar', Drabo Koine Maxim², Traoré Yves', Savadogo Aly* \\ 1 Laboratoire de Biochimie et Immunologie Appliquée, Centre de Recherche en Sciences Biologiques, Alimentaires et \\ Nutritionnelles (LABIAJCRSBAN), Université Ouaga I Pr Joseph KI-ZERBO Tel/Fax (226) 5033737303 BP 7131, \\ Ouagadougou, Burkina Faso \\ 2Laboratoire National de Santé Publique, Département de Pesticides, Contaminants et Engrais, Tel/Fax (226) 253731 \\ 31, 09BP24 Ouagadougou 09, Burkina Faso. \\ *Auteur par correspondance: Savadogo Aly, Département de Biochimie-Microbiologie /LABIA/03 BP 7021 \\ Ouagadougou, Burkina Faso Tel/Fax (226)79 385707 E-mail : alysavadogo@gmail.com
}

Original submitted in on $11^{\text {th }}$ July 2017. Published online at www.m.elewa.org on $30^{\text {th }}$ September 2017 https://dx.doi.org/10.4314/jab.v117i1.3

\section{RESUME}

Objectif: La présente étude vise à évaluer les pratiques phytosanitaires des maraîchers et à fournir une meilleure connaissance des risques liés aux pratiques de maraîchage, notamment l'utilisation des pesticides et intrants chimiques à Ouagadougou au Burkina Faso.

Méthodologie et résultats : Des enquêtes ont été réalisées du 10 mars au 08 Avril et du 18 Juillet au 06 Août 2016 auprès de 200 producteurs dans les sites de Tanghin et de Boulmiougou où se pratique une forte activité de production maraîchère. Les observations ont révélées une utilisation des pesticides non autorisés souvent destinés spécialement à d'autres cultures. L'étude a révélé une imprécision manifeste de concentration des insecticides avec une moyenne du nombre d'épandage de 9,5 . En outre, $77,88 \%$ des maraîchers ont appliqué les insecticides de Profenofos sur Ipomaea batatas (Patate douce), Lambda Cyhalothrine sur Amaranthus hybridu (Amarante), Le Manèbe sur Cleome gynandra (Gynandro)et l'emanectin benzoate sur Hibiscus sabdariffa (Oseille) respectivement dans $67 \%, 55,5 \%$, et $8,66 \%$ des cas d'utilisation détournée. Les résultats révèlent que la plupart des producteurs ( $96 \%$ à Tanghin et $87 \%$ à Boulmiougou) n'observent aucune mesure de protection lors des traitements phytosanitaires des cultures. En effet, $65 \%$ des pesticides utilisés sont classés selon l'échelle de toxicité de l'OMS et $67,5 \%$ de ses sont destinés au traitement du coton et non à des cultures maraichères.

Conclusion et application : La gestion et l'utilisation très peu rigoureuses des pesticides constatés dans ces sites pourraient constituer une menace pour le maintien de la biodiversité et de la productivité des écosystèmes naturels et de la santé des producteurs et des consommateurs. Face à ce constat, le défi de la recherche serait la détermination du niveau actuel de contamination des légumes feuilles et des eaux du barrage et celui de 
Tarnagda et al., J. Appl. Biosci. 2017 Évaluation des pratiques agricoles des légumes feuilles : le cas des utilisations des pesticides et des intrants chimiques sur les sites maraîchers de Ouagadougou, Burkina Faso.

l'État serait l'initiation de programmes de sensibilisation des producteurs par rapport à une gestion plus rigoureuse des pesticides.

Mots-clés : pratiques paysannes, cultures maraîchères, pesticides, risques, Burkina Faso

\section{Evaluation of farming practices of leafy vegetables: the case of uses of pesticides and chemical inputs in gardeners sites of Ouagadougou, BURKINA FASO. ABSTRACT}

Objective: The aim of this study was to evaluate leafy vegetable in gardeners' sites and to provide a better knowledge of the risks associated with the use of pesticides and chemical inputs.

Methods and results: Surveys were carried out at 10 march to 8 April 2016 and 18 July to 6 August 2016 with 200 producers at the Tanghin and Boulmiougou sites, where there are strong vegetable production activity.. Observations revealed the use of unauthorized pesticides, often targeted specifically for other crops. The study revealed an imprecision of the concentration of insecticides with an average application number of 9.5. In addition, $77.88 \%$ of gardeners applied Profenofos insecticides on Ipomaea batatas (Sweet potato), Lambda cyhalothrin on Amaranthus hybridus (Amaranth), Maneb on Cleome gynandra (Gynandro) and Emanectin benzoate on Hibiscus sabdariffa (Sorrel) in $67 \%, 55.5 \%$, and $8,66 \%$ of cases of misused use. The results showed that most producers (96\% in Tanghin and $87 \%$ in Boulmiougou) do not observe any protection measures during the phytosanitary treatments of crops. Indeed, $65 \%$ of pesticides are classified in the WHO toxicity scale and $67.5 \%$ of these products are destined for treatments in cotton

Conclusion and application: The very poor management and use of pesticides found at these sites could pose a threat to the maintenance of biodiversity, the productivity of natural ecosystems and the health of producers and consumers. The research challenge would be to determine the current level of contamination of leafy vegetables and dam water. That of the State would be the initiation of producer awareness programs in relation to a more rigorous management of pesticides.

Keywords: peasant practices, market gardening, pesticides, risks, Burkina Faso

\section{INTRODUCTION}

Au Burkina Faso, le souci d'augmenter la production et la productivité agricoles conduit les paysans à utiliser plus souvent les pesticides pour la lutte contre les bioagresseurs. Pour faire face à ces bioagresseurs, l'utilisation des pesticides chimiques de synthèse est la solution la plus utilisée. Cependant, plusieurs études au Burkina Faso (IFDC, 2007; Toé, 2010; Naré et al., 2015) ont souligné l'existence de mauvaises pratiques phytosanitaires: non-respect des doses prescrites, non-respect des règles de protection et d'hygiène conseillées lors des traitements, mauvaise gestion des emballages vides des pesticides. Les conséquences en sont l'intoxication des agriculteurs et des consommateurs, la pollution de l'environnement et la sélection de souches de bioagresseurs résistantes aux pesticides (Cissé et al., 2006; Toé, 2010; Ahouangninou et al., 2011; Ngom et al., 2012; Gnankiné et al., 2013; Roditakis et al., 2015; Lehmann et al., 2016a). Les légumes sont activement utilisées dans l'alimentation humaine en raison de leurs effets nutritionnels et bénéfiques sur la santé. Au Burkina Faso, ces denrées alimentaires ont trois provenances essentielles qui sont : le milieu paysan, l'importation et l'agriculture péri-urbaine. La production légumière péri-urbaine attire plus l'attention de la population urbaine car elle fait partie intégrante de la ville et fournit en toutes saisons aux citadins, des légumes frais munis de leurs qualités nutritionnelle et organoleptique (Jouve et Padilla, 2007; Kouakou et al., 2010; Olahan, 2010). Les producteurs travaillent sur des sites soumis à plusieurs contraintes sanitaires (Koffi-Nevry et al., 2012). La plupart des sites de production se trouvent dans des endroits où débouchent les canalisations chargées d'effluents domestiques (ordures ménagères) et industriels. Hormis les déchets ménagers et industriels, les maraîchers utilisent abusivement les pesticides pour 
Tarnagda et al., J. Appl. Biosci. 2017 Évaluation des pratiques agricoles des légumes feuilles : le cas des utilisations des pesticides et des intrants chimiques sur les sites maraîchers de Ouagadougou, Burkina Faso.

accroître la production légumière (Dembélé et al., 2008; Doumbia et al., 2009; Tano et al., 2011) ; ce qui peut en plus être source de contamination chimique des légumes. Toutes ces molécules chimiques libérées dans l'environnement s'infiltrent dans le sol ou ruissellent et rejoignent les écosystèmes aquatiques comme réceptacle final (Assogba-Komlan et al., 2007). Cette étude a donc été menée auprès des producteurs de légumes

\section{MATERIEL ET METHODES}

L'étude a été conduite du 10 mars au 08 Avril et du 18 Juillet au 06 Août 2016. Les entretiens avec les producteurs ont porté sur leur niveau d'instruction, les cultures pratiquées, les principaux problèmes phytosanitaires rencontrés, les méthodes de lutte utilisées, les produits phytosanitaires utilisés, le dosage, les moyens de protection utilisés, la gestion des emballages et leurs connaissances du risque chimique. Ces données ont été collectées à partir des enquêtes semi-structurées individuelles (Savoie-Zajc, 1997). Les données collectées ont fait l'objet d'un dépouillement feuilles de Tanghin et de Boulmiougou deux quartiers de la ville de Ouagadougou au Burkina Faso, pour comprendre les motifs qui déterminent l'emploi des produits chimiques dans la production de légumes feuilles et évaluer les pratiques phytosanitaires de ces producteurs en termes d'impacts potentiels sur la santé humaine et sur l'environnement.

manuel et ont été analysées à l'aide du logiciel Epi-info, version Eureka 2003. Les conditions écologiques de ses deux sites sont particulièrement favorables au développement de l'horticulture. La nappe phréatique est peu profonde avec des puits d'une profondeur variant de 3 à $7 \mathrm{~m}$ suivant la géomorphologie de la localité. Par contre, le milieu est particulièrement favorable à la prolifération des parasites ravageurs des cultures. La figure 1 nous donne la situation géographique de la zone d'étude.

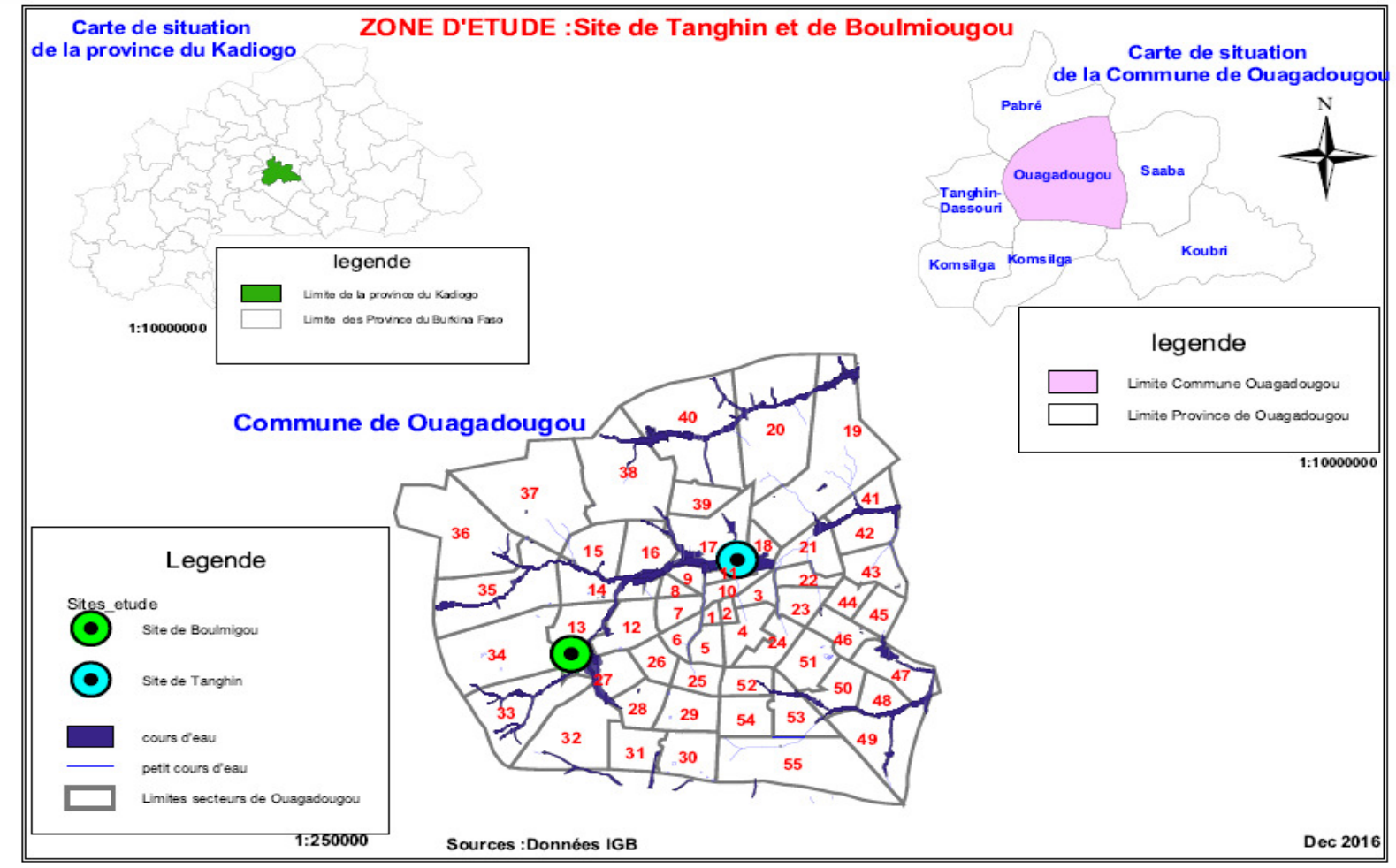

Source : Cadastre ; Réalisation : TARNAGDA et SAWADOGO, 2016.

Figure 1 : Situation géographique de la zone d'étude 
Tarnagda et al., J. Appl. Biosci. 2017 Évaluation des pratiques agricoles des légumes feuilles : le cas des utilisations des pesticides et des intrants chimiques sur les sites maraîchers de Ouagadougou, Burkina Faso.

\section{RESULTATS ET DISCUSSION}

Mode d'irrigation: Diverses techniques sont utilisées pour l'arrosage des cultures. Cette diversité est liée à la source d'alimentation en eau et au pouvoir d'achat des exploitants. Les ressources d'eau utilisées pour l'irrigation sont le barrage, les puits à faible profondeur (1 à $2 \mathrm{~m}$ ) et les tranchées creusées dans la cuvette par les maraîchers. $22 \%$ des producteurs utilisent l'eau du

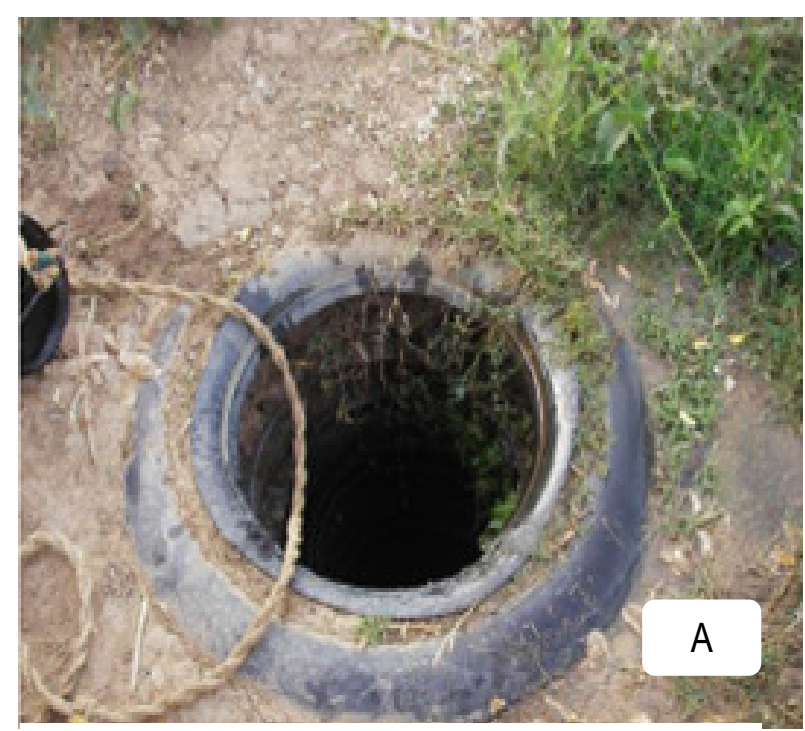

Figure 2 : Puisard protégé par des pneus au quartier Boulmiougou de Ouagadougou

Usage des mesures de protection dans l'emploi des pesticides : Dans les normes toute activité d'épandage de pesticides requiert des mesures particulières de protection. Mais, à travers cette étude, il ressort que $96 \%$ des maraîchers dans le site de Tanghin et $87 \%$ dans le site de Boulmiougou n'utilisent pas des mesures de protection et/ou ne se protègent pas durant l'activité barrage directement, $60 \%$ l'eau des puits (figure 2) uniquement et $18 \%$ utilisent les deux types de ressources (figure 3). Toutes les cultures en saison sèche se pratiquant dans le lit du barrage, la distance moyenne entre l'eau du barrage et les cultures sont estimées à $60 \pm 10 \mathrm{~m}$ avec une distance minimale de $20 \mathrm{~m}$ et une distance maximale de $500 \mathrm{~m}$.

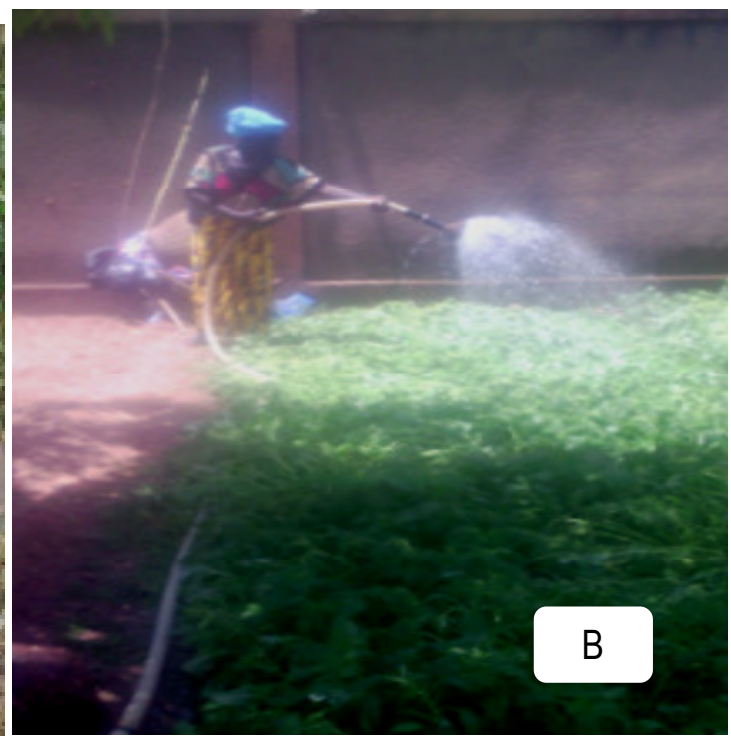

Figure 3 : Irrigation par système de motopompe + tuyaux en PVC.

d'épandage des pesticides. Très peu de maraîchers possèdent des équipements de protection adéquats ou adaptés (masques, gants, lunettes, bottes, combinaisons). Les figures 4 et 5 montrent les méthodes d'épandage avec le bidon coupé et la calebasse utilisées par les maraîchères. 


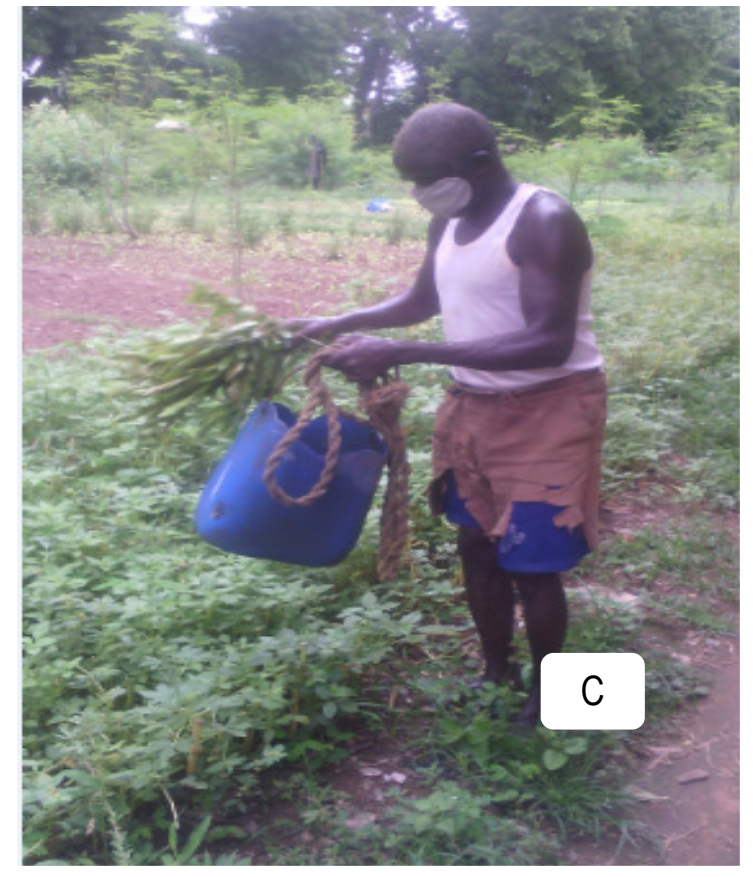

Figure 4 : Méthodes d'épandage avec un bidon coupé

Traitements phytosanitaires et délais de rémanence : Dans le site de Tanghin, les traitements phytosanitaires se font pour $75 \%$ des maraîchers, dans l'après-midi et 25 $\%$ le font le plus souvent le matin. Dans le site de Boulmiougou, la plupart des maraîchers traitent leurs cultures à tout moment de la journée (matin et soir). Ainsi, $48 \%$ des maraîchers observent un délai de carence compris entre 7 et 14 jours et $37 \%$ un délai compris entre 14 et 21 jours. Seulement $15 \%$ des maraîchères appliquent un délai de moins d'une semaine. Très peu de maraîchères $(3 \%)$ observent un délai au-delà de 21 jours. Selon la littérature la demi-vie des pyréthrinoîdes est estimée de 5 à 7 jours, quant aux carbamates elle est

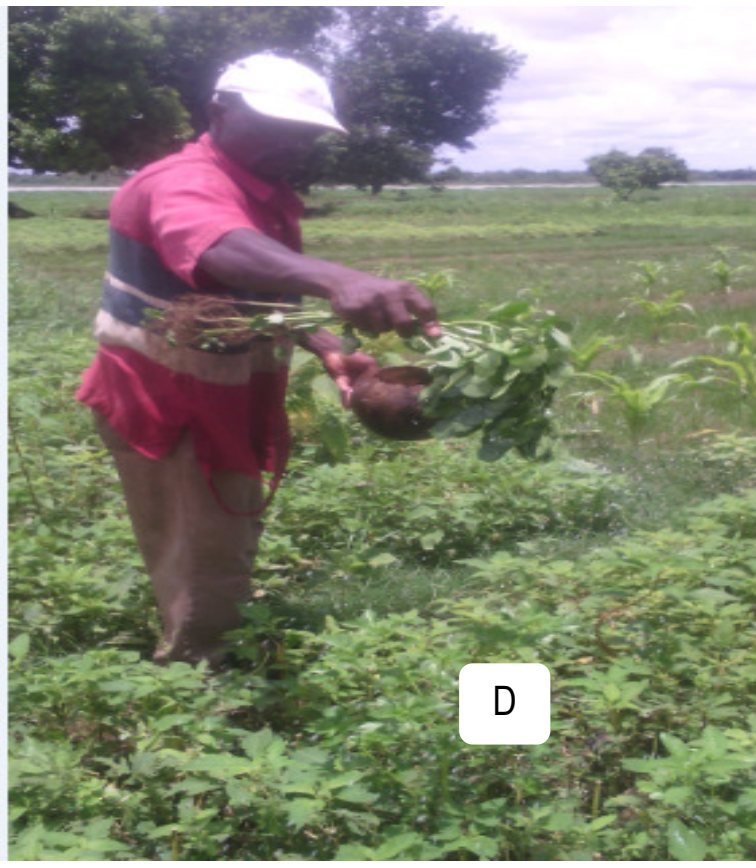

Figure 5 : Méthodes d'épandage avec une calebasse

estimée de 10 à 20 jours, pour les organophosphorés elle est estimée de 5 à 15 jours et en fin pour les organochlorés elle de 28 jours à 12,8 ans.

Méthodes de dosage du produit: Les maraîchers estiment n'avoir aucune difficulté pour doser les produits chimiques. Les pesticides utilisés sont presque tous des solutions concentrées émulsifiables (EC). Le produit est prélevé et mélangé directement à l'eau. La quantité d'eau utilisée dépend de la quantité du produit prélevée. C'est le protocole du dosage décrit par les maraîchers euxmêmes qui est utilisé. Les instruments de dosage des produits sont recensés dans la figure 6 . 


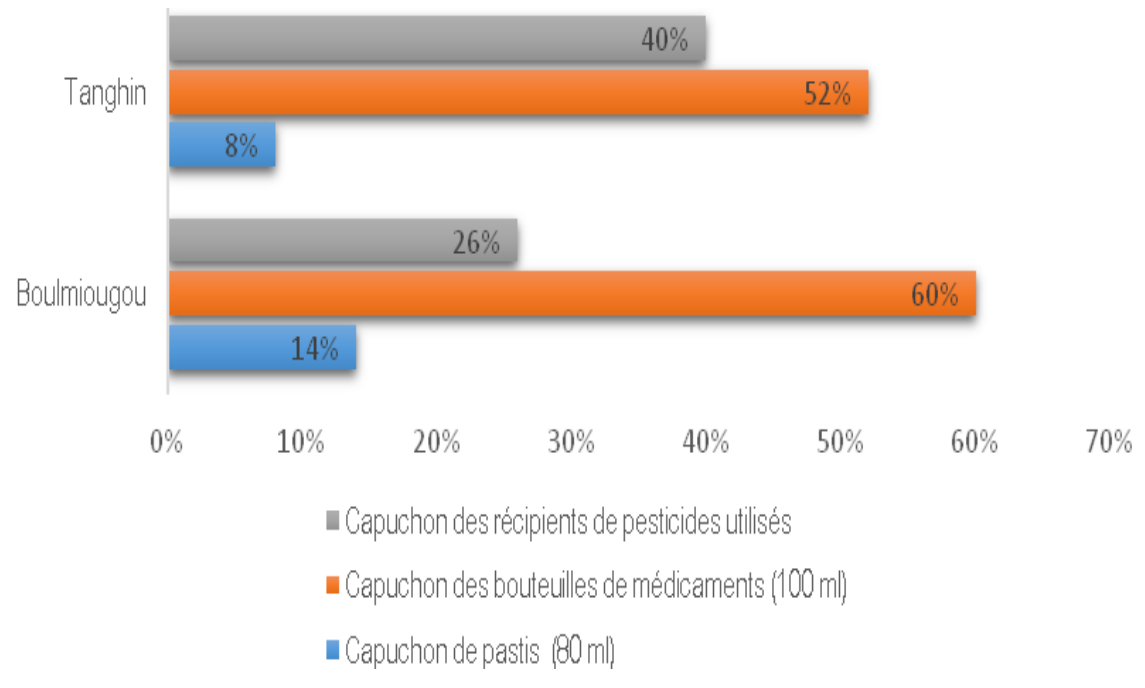

Figure 6 : Instruments de dosage des produits phytosanitaires

Ces capuchons de dosage sont imprécis parce que leurs volumes sont variables et ne sont pas gradués. Les produits utilisés peuvent être plus ou moins concentrés. Gestion des emballages: Après usage des produits phytosanitaires, $84 \%$ des maraîchères du site de Boulmiougou et $72 \%$ de celui de Tanghin jettent leurs emballages de pesticides dans la nature. Ce qui augmente le risque de contamination de l'environnement. Cette forme de recyclage des emballages de produits phytosanitaires est due au fait que les maraîchères sont très peu informés des risques écologiques encourus par la mauvaise gestion de ces emballages.

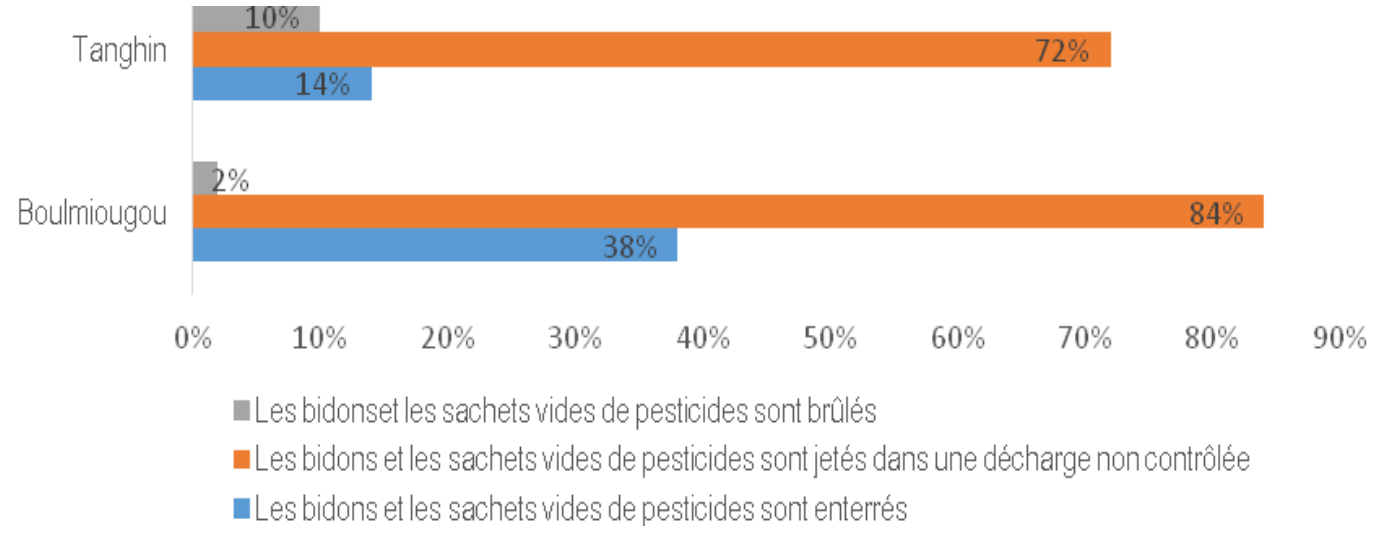

Figure 7 : Gestion des emballages

Caractéristiques sociodémographiques: Les caractéristiques sociodémographiques prises en compte dans cette enquête étaient le sexe, l'âge et le niveau d'instruction des maraîchers. Ces caractéristiques, en particulier le niveau d'instruction, peuvent influencer grandement les pratiques d'utilisation des produits phytosanitaires. Les résultats obtenus pour ces différents paramètres sont présentés dans le Tableau 1. 
Tarnagda et al., J. Appl. Biosci. 2017 Évaluation des pratiques agricoles des légumes feuilles : le cas des utilisations des pesticides et des intrants chimiques sur les sites maraîchers de Ouagadougou, Burkina Faso.

Tableau 1 : Caractéristiques sociodémographiques de la population étudiée

\begin{tabular}{cccc}
\hline & & \multicolumn{2}{c}{ Fréquences (\%) } \\
\hline Variables & Catégories & Tanghin & Boulmiougou \\
\hline Age (ans) & $15-35$ & 36 & 50 \\
& $36-55$ & 54 & 42 \\
& 56 et plus & 10 & 8 \\
& Total & 100 & 100 \\
& & & \\
Éducation & Sans éducation & 65 & 75 \\
& Primaire & 25 & 20 \\
& Secondaire & 10 & 5 \\
& Supérieurs & 0 & 0 \\
& Total & 100 & 100 \\
\hline
\end{tabular}

Niveau de perception : Les informations recueillies dans le site de Tanghin et de Boulmiougou à propos des pesticides, de même que des nuisibles combattus, la fréquence des produits, la catégorie de produits, le mode d'acquisition, la classification selon OMS, modes d'usage et l'aspect sont consignées dans le Tableau 2. 
Tableau 2: Pesticides utilisés sur le site de Tanghin et de Boulmiougou

\begin{tabular}{|c|c|c|c|c|c|c|c|c|}
\hline & & & & & & & Site & d'étude \\
\hline $\begin{array}{c}\mathrm{Fr} \\
\left(\mathrm{ni} / \mathrm{N}^{*}\right. \\
100)\end{array}$ & Nom commercial & Molécules actives & $\begin{array}{c}\text { Nuisibles importants } \\
\text { traités }\end{array}$ & $\begin{array}{l}\text { Catégorie des } \\
\text { produits }\end{array}$ & Application & $\begin{array}{c}\text { Classificati } \\
\text { on } \\
\text { OMS } \\
\end{array}$ & Tanghin & Boulmiougou \\
\hline 25,8 & CAÏMAN ou PROTECT(f) & $\begin{array}{c}\text { Emamectine benzoate } \\
19,2 \mathrm{~g} / \mathrm{L}\end{array}$ & Pucerons ; & Insecticide & Coton & II & + & + \\
\hline 9,67 & Néant (Tikaame: Mooré) & Adonis; Pyrical 480 UL & Insectes & Insecticide & Maraîchage & II & + & + \\
\hline 41,93 & $\mathrm{D} 6^{(\mathrm{b})}$ & Deltaméthrine $15 \mathrm{~g} / \mathrm{L}$ & Insectes & Insecticide & Maraîchage & $\|$ & + & - \\
\hline 9,67 & ACARICE 018 EC(b) & Abamectine $18 \mathrm{~g} / \mathrm{L}$ & Pucerons & Insecticide & Coton & $\|$ & + & + \\
\hline 9,67 & DELTA CAL (12,5 EC) (b) & Deltaméthrine & Chenilles dévoreuses & Insecticide & Maraîchage & ॥ & + & + \\
\hline 16,13 & $\begin{array}{l}\text { LAMBDA SUPER 2,5 } \\
\qquad \mathrm{Ec}^{(\mathrm{b})}\end{array}$ & Lambda Cyalothrin & Tordeuses de feuilles & Insecticide & $\begin{array}{l}\text { Coton/ } \\
\text { Maraîchage }\end{array}$ & $\mathrm{lb}$ & + & - \\
\hline 35,48 & CONQUEST C176 Ec(b) & $\begin{array}{l}\text { Cypermétrine } 72 \mathrm{~g} / \mathrm{L} \\
\text { acétamipride } 16 \mathrm{~g} / \mathrm{L}\end{array}$ & $\begin{array}{l}\text { Mouches blanches ; } \\
\text { pucerons }\end{array}$ & Insecticide & Coton & III & + & + \\
\hline 61,29 & ATTA CK(f) & Emamectine benzoate & $\begin{array}{l}\text { Chenilles rongeuses } \\
\text { de feuilles ; pucerons }\end{array}$ & Insecticide & Coton & II & + & + \\
\hline 3,22 & EMIR FORT 104 Ec (b) & $\begin{array}{l}\text { Cypermétrine } 72 \mathrm{~g} / \mathrm{L}+ \\
\text { acétamipride } 32 \mathrm{~g} / \mathrm{L}\end{array}$ & $\begin{array}{c}\text { Mouches blanches ; } \\
\text { pucerons }\end{array}$ & Insecticide & Coton & $\|$ & + & + \\
\hline 16,13 & TITAN 25EC(b) & Acétamipride (10 g/L) & Pucerons & Fongicide & Coton & II & + & - \\
\hline 6,45 & D-BAN SUPER(c) & Chlorpyriphos & $\begin{array}{c}\text { Chenilles rongeuses } \\
\text { de feuille }\end{array}$ & Insecticide & Maraîchage & II & + & + \\
\hline 22,58 & SAVAHALER(d) & Méthomyl 250g/kg & Insectes & Insecticide & Coton & lb & + & - \\
\hline 3,22 & FURADAN(d) & Carbofuran $50 \mathrm{~g} / \mathrm{Kg}$ & Insectes et nématodes & Insecticide & Maraîchage & $\mathrm{lb}$ & + & + \\
\hline 9,67 & RAMBO (Pif-Paf) (b) & Permethrin 0,60\% & Insectes & Insecticide & Maraîchage & $\|$ & - & - \\
\hline 3,22 & $\operatorname{IBISA}(\mathrm{a})$ & $\begin{array}{c}\text { Alpha-cyperméthrine + } \\
\text { acétamipride }\end{array}$ & Champignons & Fongicide & Coton & $\|$ & - & + \\
\hline 9,67 & THEORAL (c) & $\begin{array}{c}\text { Chlorpyrifos-éthyl } 25 \%+ \\
\text { Thirame }\end{array}$ & $\begin{array}{l}\text { Pucerons (bruns, } \\
\text { noirs) }\end{array}$ & Insecticide & $\begin{array}{l}\text { Coton/ } \\
\text { Maraîchage }\end{array}$ & $\|$ & + & + \\
\hline 7,31 & TANGO 500 EC(c) & Profénofos & Chenilles & Insecticide & Coton & III & - & + \\
\hline 2,44 & ALMANE B 80 WP(e) & Manèbe $80 \%$ & Chenilles & Fongicide & Coton & U & - & + \\
\hline 7,31 & DUEL 186 EC(b) & $\begin{array}{c}\text { Cyperméthrine } 39 \mathrm{~g} / \mathrm{L} \\
\text { Ec }\end{array}$ & Insectes & Insecticide & Maraîchage & III & + & + \\
\hline
\end{tabular}

NB : (a) Association de pesticides, ${ }^{(b)}$ Pyréthrinoïde ; ${ }^{(c)}$ Organophosphoré ; ${ }^{(d)}$ Carbamate ; ${ }^{(e)}$ Dithiocarbamates ; ${ }^{(f)}$ Organochloré. la = Extrêmement dangereux; Ib = Hautement dangereux; II = Modérément dangereux; III = Légèrement dangereux; U = Ne semble pas présenter de danger dans les conditions normales d'utilisation; Effectifs $(N)=$ Cent (200) maraîchers 

utilisations des pesticides et des intrants chimiques sur les sites maraîchers de Ouagadougou, Burkina Faso.

\section{DISCUSSION}

Cette étude montre que les pratiques d'utilisation des pesticides sont identiques au Burkina et dans de nombreux autres pays d'Afrique (Cissé et al., 2008; Ngom et al., 2009; Diop, 2013). L'activité maraichère dans les deux (02) sites étudiés mobilise une population à dominance jeune (15-35 ans). Cette situation corrobore celle décrite par Diop (2013). Des résultats différents ont été obtenus au Nigéria où la classe d'âge 26-35 ans ne représentaient que 9,6\% (Banjo et al., 2010) et au Botswana avec $24,1 \%$ des exploitants agricoles âgés entre 30 et 40 ans (Obopile et al., 2008). Les résultats obtenus dans ce travail, sont en corrélation avec ceux obtenues par Congo (2013) qui ont montré que 87,92 \% des maraîchers ont moins de cinquante (50) ans. En plus, le nombre élevé de ceux ayant plus de cinquante (50) ans, se révèle très préoccupant quand on sait que la capacité fonctionnelle de certains organes vitaux baisse avec l'âge (Toé, 2010). II en résulte une augmentation des risques sanitaires liés à l'exposition aux pesticides due à l'inaptitude de l'organisme à éliminer les xénobiotiques après leur intrusion en son sein du fait de l'âge. L'absence de qualification professionnelle combinée à un taux de chômage élevé offre des opportunités de travail limitées à la jeunesse. Ainsi, ils s'engagent dans ce secteur qui ne nécessite souvent pas de qualification tel que le montre le taux d'analphabétisme élevé chez les maraîchers de nos zones d'étude ( $65 \%$ à Tanghin et $75 \%$ à Boulmiougou). Des résultats similaires ont été trouvés au Nigéria par Banjo et al. (2010) et au Burkina Faso par Congo, 2013. Ces auteurs ont montré que la majorité des maraîchers enquêtés sont analphabètes (soit $77,18 \%$ ) et les $22,82 \%$ restant possèdent un niveau insuffisant de compréhension. Les individus à faible niveau d'instruction ont des difficultés à trouver un emploi dans le secteur formel, ce qui justifie en partie leur forte représentativité dans le maraîchage (Diop, 2013) où l'activité n'exige pas de compétences spécifiques. Ces résultats sont superposables à ceux rapportés par Ntow et al. (2006) au Ghana où tous les répondants utilisaient des pesticides et à ceux de Sibanda et al. (2000) au Zimbabwé, Ngom et al. (2012) au Sénégal et par Congo (2013) au Burkina Faso. Plusieurs raisons permettent d'expliquer cette situation: disponibilité de produits abordables en sachets unidosés ou conditionnés dans des flacons sur le marché informel, culture de légumes sensibles aux attaques, perceptions des risques potentiels et des pertes de rendement par les maraîchers, absence d'autres alternatives de gestion des ravageurs immédiatement disponibles (Williamson et al., 2008; Congo, 2013). Au total, 20 matières actives et associations de matières actives ont été citées par les maraîchers. Ce chiffre est proche de celui rapporté précédemment dans d'autres sites à Loumbila (Congo, 2013) au Burkina Faso; Sène (2008) au Sénégal et de celui trouvé par Sibanda et al. (2000) au Zimbabwé. Dans la présente étude, l'insecticide était les pesticides les plus représentés tandis que les fongicides n'étaient représentés que par l'alpha-cyperméthrine + acétamipride et le manèbe, un pesticide de la famille des dithiocarbamates. Ces résultats sont différents de ceux retrouvés au Ghana par Ntow et al. (2006), où ce groupe était le plus représenté. Quatre pesticides appartenant aux classes la et lb de I'OMS sont appliqués sur les cultures à des fréquences élevées (33\% sur le site de Tanghin et $36 \%$ site de Boulmiougou) (Tableau 2). Des résultats similaires ont été rapportés du Sénégal par Diop (2013) du Botswana par Obopile et al. (2008) où près du tiers des matières actives utilisées par les paysans étaient classées comme extrêmement ou hautement dangereuses. Les maraîchers se protègent très peu au moment des traitements à l'instar des études menées ailleurs (Ahouangninou et al., 2011). Selon Doumbia et Kwadjo (2009), I'utilisation de protection lors des traitements n'est pas un critère de différenciation des maraîchers même si beaucoup de producteurs utilisent une protection minimale lors de l'épandage des pesticides. $99,5 \%$ des producteurs dans notre étude ont utilisé des tenues simples, qui étaient soit une vieille chemise déchirée et trouée avec des manches courtes, soit des vieux pantalons courts, déchirés ou troués sans masques, ni gants, ni bottes ou chapeau de protection. $45 \%$ des maraîchers qui se protègent utilisent seulement des cache-nez ou des foulards chez les femmes. Selon Sougnabe et al. (2009); Congo (2013) et Diop (2013), les maraîchers ne se protègent pas en raison du coût élevé du matériel de protection. Or, il a été montré que le manque de matériels de protection corporelle accroît les risques d'intoxication qui, mineurs au début, peuvent devenir graves par bioaccumulation ( INRS, 2007; Gomgnimbou et al., 2009). Des constatations similaires ont été notées au Nigéria par Banjo et al., (2010) ; au Burkina Faso par Congo (2013) et au Sénégal par Ngom et al. (2012). Pour mesurer les quantités de pesticide à appliquer, les maraîchers utilisent les capuchons des flacons des pesticides, ces flacons ou plus rarement une cuillère à café ou la balance. 
Tarnagda et al., J. Appl. Biosci. 2017 Évaluation des pratiques agricoles des légumes feuilles : le cas des utilisations des pesticides et des intrants chimiques sur les sites maraîchers de Ouagadougou, Burkina Faso.

\section{CONCLUSION}

Cette étude a permis de mieux appréhender les différentes pratiques culturales à risques liés à l'utilisation des produits phytosanitaires dans deux sites et d'en tirer les conséquences pour l'environnement et la santé humaine. II ressort des résultats obtenus que les pratiques d'utilisation des pesticides sont peu respectueuses des normes de protection sanitaires aussi des utilisateurs que de l'écosystème environnant. La méconnaissance et le non-respect des directives pour le dosage des pesticides, l'utilisation des pesticides du cotonnier sur les cultures maraîchères, l'absence d'utilisation des équipements de protection sanitaire font partie entre autres des mauvaises pratiques culturales.

\section{REMERCIEMENTS}

Cette étude a été réalisée avec le soutien financier de la Banque Islamique Développement (BID).

\section{RÉFÉRENCES BIBLIOGRAPHIQUES}

Ahouangninou C, Fayomi B. E, Martin T, 2011. Evaluation des risques sanitaires et environnementaux des pratiques phytosanitaires des producteurs maraîchers dans la commune rurale de Tori-Bossito (Sud-Bénin),. Cahier Agriculture., 20(3), 216-222.

Assogba-Komlan F, Anihouvi P, Achigan E, Sikirou R, Boko A, Adje C, Vodouhè R. et Assa A, 2007. Pratiques culturales et teneur en éléments anti nutritionnels (nitrates et pesticides) du Solanum macrocarpum au sud du Bénin. African Journal of Food Agriculture Nutrition and Development, 7(4), 1-21.

Banjo A D, Aina S A, Rije O I, 2010. «FFarmers' knowledge and perception towards herbicides and pesticides usage in Fadama area of OkunOwa, Ogun State of Nigeria,». African Journal of Basic and Applied Sciences, 2, 188-194.

Cissé G, Matthys B, Adiko A F, 2006. Le réseau social des maraîchers à Abidjan agit sur la perception des préoccupations et des risques sanitaires liés à l'eau, Vertigo -. La Revue Électronique En Sciences de L'environnement, Hors-Série 3.

Cissé I, Badiane B, Ngom S, Diop YMB, Séne M, 2008. Usage des pesticides et risques sanitaires sur la production horticole de la zone des Niayes au Sénégal,. Rev. Sn. Res. Agric., Vol. 1(3), 19-26.

Congo K, 2013. Risques sanitaires associes à l'utilisation de pesticides autour de petites retenues : cas du barrage de loumbila. In Mémoire master 2iE ( $\mathrm{p}$. 68).

Dembélé A, Oumarou B, Traore SK, Mamadou K, Coulibaly DT, AmadouT, 2008. The chemical control of the pests in the truck farming and the quality of vegetables in african urban cities: the health hazards and security of consumers. European Journal of Scientific Research, 20, 836-843.
Diop A, 2013. Diagnostic des pratiques d'utilisation et quanti cation des pesticides dans la zone des Niayes de Dakar (Sénégal). In Thèse de doctorat Chimie analytique (p. 241).

Doumbia M, Kwadjo. KE, 2009. Pratiques d'utilisation et de gestion des pesticides par les maraîchers en Côte d'Ivoire : Cas de la ville d'Abidjan et deux de ses banlieues (Dabou et Anyama). Journal of Applied Biosciences, 18, $992-1002$.

Gnankiné O, Mouton L, Savadogo A, Martin T, Sanon A, Dabiré $R, 2013$. Biotype status and resistance to neonicotinoids and carbosulfan in Bemisia tabaci (Hemiptera : Aleyrodidae) in Burkina Faso, West Africa. International Journal of Pest Management 59, (2), 95-102.

Gomgnimbou APK, Savadogo PW, Nianogo AJ, MillogoRasolodimby J, 2009. Usage des intrants chimiques dans un agrosystème tropical: diagnostic du risque de pollution environnementale dans la région cotonnière de l'est du Burkina Faso,. Biotechnol. Agron. Soc. Environ., 13(4), 499-507.

IFDC, 2007. Problématique de l'utilisation des produits phytosanitaires en conservation des denrées alimentaires et en maraîchage urbain et périurbain au Burkina Faso: cas de Bobo Dioulasso, Ouahigouya et Ouagadougou. Ouagadougou/Burkina Faso : IFDC., 51.

INRS, 2007. Le mercure-prévention de l'hydrargyrisme,. Jouve AM, Padilla M, 2007. Les agricultures périurbaines méditerranéennes à l'épreuve de la multifonctionnalité: comment fournir aux villes une nourriture et des paysages de qualité ? Cahiers Agricultures, 16, 311-317.

Koffi-Nevry R., Assi-Clair BJ, Assemand EF, Affou SW, Koussemon M, 2012. Origine des témoins de contamination fécale de l'eau d'arrosage de la laitue (Lactuca sativa) cultivée dans la zone 
Tarnagda et al., J. Appl. Biosci. 2017 Évaluation des pratiques agricoles des légumes feuilles : le cas des utilisations des pesticides et des intrants chimiques sur les sites maraîchers de Ouagadougou, Burkina Faso.

périurbaine d'Abidjan. J. of Applied Biosciences, 52, 3669-3675.

Kouakou YE, Koné B, Bonfoh B, Kientga SM, N'Go YA, Savane I, Cissé G, 2010. L'étalement urbain au péril des activités agro-pastorales à Abidjan. Vertigola revue électronique en sciences de l'environnement, , consulté le 10 décembre 2015. Hors-Série 3, 10(2).

Lehmann E, Oltramare C, Nfon DJJ, Konaté Y, De Alencastro L, 2016. Assessment of occupational exposure to pesticides with multi-class pesticide residues analysis in human hairs using a modified QuEChERS extraction method, case study of gardening areas in Burkina Faso. In: Annual Meeting of the International Association of Forensic Toxicologists (TIAFT), Brisbane, Australia.

Naré RWA, Savadogo PW, Gnankambary Z, Nacro HB, Sedogo M, 2015. Analyzing risks related to the use of pesticides in vegetable gardens in Burkina Faso. Agriculture, Forestry and Fisheries, 4(4), 165-172.

Ngom S, Traore S, Thiam MB, Anastasie M, 2012. Contamination des produits agricoles et de la nappe phréatique par les pesticides dans la zone des Niayes au Sénégal. Rev. Sci. Technol., Synthèse, 25, 12.

Ngom S, Cissé I, Traore S, Rousseau J, Anastasie M, 2009. Incidence des pesticides sur la qualité des ressources en eaux et des produits agricoles dans la zone des Niayes au Sénégal,. In 2ème Colloque francophone en environnement et santé, Université Badji Mokhtar de Annaba, Algérie.

Ntow WJ, Gijzen HJ, Kelderman P et P. D. 2006. «Farmer perceptions and pesticide use practices in vegetable production in Ghana,». Pest Manage. Sci. , 62, 356-365.

Obopile M, Munthali DC et Matilo B, 2008. «Farmers' knowledge, perceptions and management of vegetable pests and diseases,». Crop Prot., 27, 1220-1224.
Olahan A, 2010. Agriculture urbaine et stratégies de survie des ménages pauvres dans le complexe spatial du district d'Abidjan. Vertigo, VertigO La Revue Électronique En Sciences de L'environnement, 10(2).

Roditakis E, Vasakis E, Grispou M, Stavrakaki M, Nauen $R$, Gravouil M, 2015. First report of Tuta absoluta resistance to diamide insecticides. Journal of Pest Science, 88, 9-16.

Savoie-Zajc L, 1997. L'entrevue semi-dirigée. Dans B. Gautier, Recherche sociale: de la problématique à la collecte des données SteFoy : Presses de l'Université du Québec, 23.

Sène $M, 2008$. Pratiques d'utilisation des pesticides et teneurs en résidus des produits horticoles dans la zone des Niayes de Mboro: effets éventuels sur la santé publique. In Thèse de pharmacie, Dakar: UCAD,.

Sibanda T, Dobson HM, Cooper JF, Manyangarirwa W, Chiimba W, 2000. «Pest management challenges for smallholder vegetable farmers in Zimbabwe,». Crop Prot, 19, 807-815.

Sougnabe Souapibe Pabame, Abel Yandia, Joseph Acheleke, Thierry Brevault, Sayre MV, 2009. Pratiques phytosanitaires paysannes dans les savanes d'Afrique centrale. In Savanes africaines en d_eveloppement: innover pour durer, (p. 13).

Tano BF, Abo K, Dembélé A, Fondio L, 2011. Systèmes de production et pratiques à risque en agriculture urbaine: cas du maraîchage dans la ville de Yamoussoukro en Côte d'lvoire. International Journal of Biological and Chemical Sciences, 5, 2317-2329.

Toé A, 2010. Etude pilote des intoxications dues aux pesticides agricoles au Burkina Faso.

Williamson S, Ball A, Pretty J, 2008. «Trends in pesticide use and drivers for safer pest management in four African countries,». Crop Prot, 27, 13271334. 